
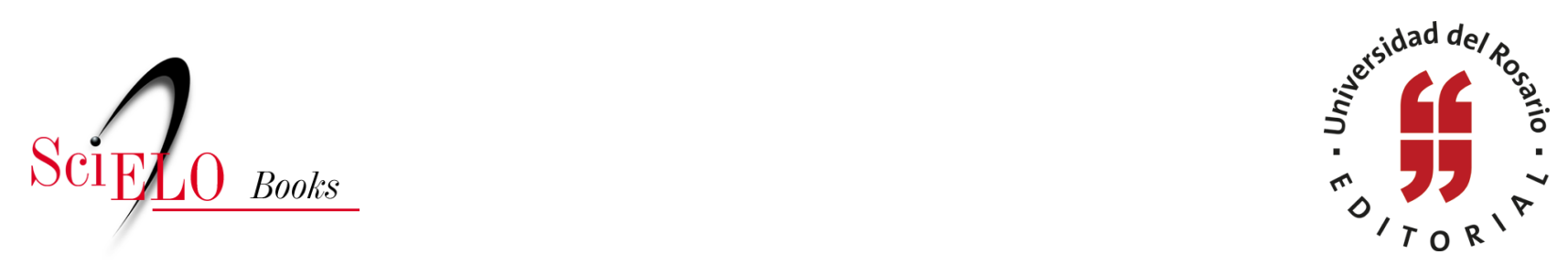

\title{
Capítulo 1. Estratificación socioeconómica y la información catastral. \\ Introducción al problema y perspectivas a futuro
}

\author{
Juan Miguel Gallego \\ Denis López \\ Carlos E. Sepúlveda
}

\section{SciELO Books / SciELO Livros / SciELO Libros}

GALLEGO, J.M., LÓPEZ, D., and SEPÚLVEDA, C.E. Estratificación socioeconómica y la información catastral. Introducción al problema y perspectivas a futuro. In: SEPÚLVEDA RICO, C.E., LÓPEZ CAMACHO, D., and GALLEGO ACEVEDO, J.M., eds. Los límites de la estratificación: en busca de alternativas [online]. Bogotá: Editorial Universidad del Rosario: Alcaldía Mayor de Bogotá D.C., 2014, pp. 1-21. ISBN: 978-958-738-537-3. https://doi.org/10.7476/9789587385373.0003.

\section{International license.}

Todo o conteúdo deste trabalho, exceto quando houver ressalva, é publicado sob a licença Creative Commons Atribição 4.0.

Todo el contenido de esta obra, excepto donde se indique lo contrario, está bajo licencia de la licencia $\underline{\text { Creative }}$ Commons Reconocimento 4.0 . 


\title{
Capítulo 1 Estratificación socioeconómica y la información catastral. Introducción al problema y perspectivas a futuro
}

\author{
Juan Miguel Gallego", Denis López y Carlos E. Sepúlveda***
}

\section{Introducción}

Este libro busca proponer alternativas a las diagnosticadas deficiencias instrumentales del sistema actual de estratificación socioeconómica en la ciudad de Bogotá, D.C., y profundiza en cómo la falta de actualización metodológica y de contextualización de la dinámica de evolución de los predios y viviendas, en particular en las zonas urbanas, afecta la efectividad del sistema en identificar adecuadamente hogares sujetos a subsidios o contribuciones de tarifas de servicios públicos domiciliarios. De igual forma, explica la forma en que instrumentos alternativos como la información catastral han evolucionado, convirtiéndose en una herramienta idónea para generar una clasificación socioeconómica que reemplace el mecanismo de estratificación socioeconómica actual.

\footnotetext{
* Profesor asociado, Facultad de Economía de la Universidad del Rosario.

** Consultor asociado Infométrika, SAS.

*** Profesor principal, Facultad de Economía de la Universidad del Rosario.
} 
La estratificación socioeconómica en Colombia se torna en un instrumento de política pública utilizado en la definición de subsidios cruzados sobre las tarifas de servicios públicos domiciliarios (SPD) de manera estándar a principios de la década de los ochenta con los diseños tarifarios de la Junta Nacional de Tarifas (JNT) ${ }^{1}$. Desde inicios de los noventa, se buscó normalizar a través de una sola metodología la forma de definir los estratos socioeconómicos y que la metodología, en su totalidad, no fuera potestad de los alcaldes, lo que se vería consignado en el capítulo IV de la Ley 142 de 1994, logrando incorporar una metodología nacional que sería definida por el gobierno central y adoptada por los municipios.

La Ley 142 de 1994 consolidó un sistema cruzado de subsidios y contribuciones a través de la definición de seis grupos a partir de características de los bienes inmuebles, de manera que se diferencie el pago de SPD entre 'estratos' socioeconómicos. Este sistema se fundamenta en los principios de solidaridad, redistribución de ingresos, simplificación y transparencia, y en preservar la viabilidad financiera de las empresas prestadoras de SPD. El modelo implementó también niveles de consumo básico o de 'subsistencia' para los usuarios de menor capacidad económica, con el objeto de enfocar los subsidios en dichos niveles de consumo.

De este modo, la estratificación socioeconómica de los inmuebles se convirtió en un instrumento de política pública, atípico en el escenario internacional, y ha sido utilizado en Colombia como herramienta central en la política de SPD. En sus inicios, jugó un papel fundamental en el acceso universal de servicios como energía y acueducto en ciudades como Bogotá, Medellín y Cali, e, igualmente, permitió alcanzar niveles importantes de cobertura para las zonas urbanas del país, facilitando a su vez el consumo de servicios en SPD a hogares con muy baja capacidad de pago. Este éxito inicial ayudó a que la estratificación evolucionara como un referente de clasificación de inmuebles (e indirectamente de hogares) y a que su uso se extendiera al utilizarse para focalizar gasto público en políticas sociales diferentes a los SPD, como, por ejemplo, subsidios a la educación superior.

A pesar de su innegable utilidad y pertinencia en el sistema de SPD, particularmente en momentos en que fuentes alternativas de información

1 La JNT se creó mediante Decreto 3069 de diciembre de 1968, que fuera publicado el 21 de enero de 1969 y que operó como parte del Departamento Nacional de Planeación. 
no estaban disponibles, el mecanismo actual de estratificación ha mostrado también importantes limitaciones en su implementación. Particularmente, la metodología de estratificación evidencia rigideces en responder de manera sostenible a una evolución urbana y socioeconómica cada vez más dinámica y compleja. Varios elementos ilustran este punto: la metodología definida no está suficientemente blindada a influencia política (e. g., presión ciudadana que conlleva un costo político de actualizarla); se evidencia dificultad de presentar actualizaciones metodológicas que se adapten a la evolución urbanística y social de ciertas ciudades (no ha habido una mejora metodológica sustancial desde 1994); esto mismo ha hecho que diferentes políticas públicas sociales que se basaban en la estratificación como instrumento de focalización hayan migrado a opciones que se desligan de los estratos a medida que se dispone de información alternativa y mejor (e. g., Sistema de Identificación de Potenciales Beneficiarios de Programas Sociales SISBEN III); la estructura normativa actual restringe la posibilidad de diseño de políticas complementarias que respondan a realidades complejas específicas de una ciudad con las características de la capital colombiana (e. g., proyectos de renovación urbana que no lleven a la gentrificación ante la presión de pagos más altos por actualización de estratos de zonas mejoradas).

Dentro de las consecuencias de la falta de actualización de las metodologías, se tiene un balance deficitario con un costo fiscal para la ciudad y el país importante. Cálculos de los autores muestran que, para 2012, el déficit nacional del sistema de solidaridad para el país ascendía a 2 billones de pesos, especialmente por un desbalance entre subsidios y contribuciones en el servicio de energía ${ }^{2}$. Para Bogotá, el déficit de todos los servicios asciende a más de 93 mil millones de pesos.

Este diagnóstico llevó a la Secretaría Distrital de Planeación (SDP) a plantear un estudio que evalúe la relación de las condiciones sociales de los hogares de la capital con la estratificación socioeconómica vigente y con la información catastral, y que analice las consecuencias de considerar el avalúo catastral como fundamento para definir modelos de estratificación de viviendas o, incluso, pensar en la posibilidad de una clasificación continua de hogares sin necesidad de estratificar o predeterminar estratos. El equipo

2 El balance negativo de energía se profundizó al eliminar las contribuciones del sector industrial en 2009. 
Tabla 1.1. Subsidios y contribuciones a nivel nacional

(Millones de \$COP - diciembre 2013)

\begin{tabular}{l|r|r|r|r|r|r}
\hline \multirow{2}{*}{$\begin{array}{c}\text { Servicio público } \\
\text { domiciliario }\end{array}$} & \multicolumn{4}{|c|}{2008} & \multicolumn{3}{c}{2012} \\
\cline { 2 - 7 } & \multicolumn{1}{|c|}{ Subsidios } & Contribuciones & Balance & \multicolumn{1}{c}{ Subsidios } & Contribuciones & \multicolumn{1}{c}{ Balance } \\
\hline Acueducto & $\$ 408.874$ & $\$ 242.586$ & $(\$ 166.288)$ & $\$ 451.801$ & $\$ 262.573$ & $(\$ 189.228)$ \\
\hline Alcantarillado & $\$ 267.871$ & $\$ 188.914$ & $(\$ 78.957)$ & $\$ 281.212$ & $\$ 197.477$ & $(\$ 83.735)$ \\
\hline Aseo & $\$ 11.434$ & $\$ 270.149$ & $(\$ 141.285)$ & $\$ 750.157$ & $\$ 375.927$ & $(\$ 374.230)$ \\
\hline Energía eléctrica & $\$ 1.341 .750$ & $\$ 1.114 .635$ & $(\$ 227.115)$ & $\$ 1.711 .234$ & $\$ 551.204$ & $(\$ 1.160 .030)$ \\
\hline Gas natural & $\$ 204.405$ & $\$ 56.162$ & $(\$ 148.243)$ & $\$ 315.996$ & $\$ 65.051$ & $(\$ 250.946)$ \\
\hline Total & $\$ 2.634 .334$ & $\$ 1.872 .446$ & $(\$ 761.888)$ & $\$ 3.510 .400$ & $\$ 1.452 .231$ & $(\$ 2.058 .169)$ \\
\hline
\end{tabular}

Fuente: SUI. Cálculo de los autores. Valores deflactados con IPC.

Tabla 1.2. Subsidios y contribuciones en Bogotá

(Millones de \$COP - diciembre 2013)

\begin{tabular}{l|r|r|r|r|r|r}
\hline & \multicolumn{3}{|c|}{2008} & \multicolumn{2}{c}{2012} \\
\hline $\begin{array}{c}\text { Servicio público } \\
\text { domiciliario }\end{array}$ & Subsidios & Contribuciones & Balance & Subsidios & Contribuciones & Balance \\
\hline Acueducto & $\$ 133.235$ & $\$ 104.107$ & $\mathbf{( \$ 2 9 . 1 2 8 )}$ & $\$ 150.655$ & $\$ 109.885$ & $(\$ 40.770)$ \\
\hline Alcantarillado & $\$ 7.443$ & $\$ 66.367$ & $\mathbf{( \$ 9 . 0 7 6 )}$ & $\$ 90.375$ & $\$ 73.913$ & $(\$ 16.462)$ \\
\hline Aseo & $\$ 90.261$ & $\$ 61.328$ & $\mathbf{( \$ 2 8 . 9 3 3 )}$ & $\$ 62.062$ & $\$ 56.165$ & $\mathbf{( \$ 5 . 8 9 7 )}$ \\
\hline Energía eléctrica & $\$ 156.373$ & $\$ 260.109$ & $\$ 103.737$ & $\$ 207.122$ & $\$ 219.524$ & $\$ 12.401$ \\
\hline Gas natural & $\$ 51.713$ & $\$ 22.618$ & $\mathbf{( \$ 2 9 . 0 9 5 )}$ & $\$ 72.417$ & $\$ 29.499$ & $(\$ 42.919)$ \\
\hline Total & $\$ 507.024$ & $\$ 514.530$ & $\$ 7.505$ & $\$ 582.632$ & $\$ 488.985$ & $(\$ 93.647)$ \\
\hline
\end{tabular}

Fuente: SUI. Cálculo de los autores. Valores deflactados con IPC.

de investigación, formado por expertos estadísticos, ingenieros catastrales, sociólogos y economistas, utilizó la riqueza estadística del catastro de Bogotá y la disponibilidad de las encuestas de hogares, como la Encuesta multipropósito de Bogotá 2011 (EMB 2011), para poder ahondar en el tema.

En este sentido, se analizan las debilidades técnicas de la estratificación como herramienta de focalización de subsidios cruzados considerando los siguientes aspectos. En primer lugar, su efectividad como herramienta para 
identificar y clasificar grupos de hogares/viviendas ${ }^{3}$ diferenciados en sus condiciones socioeconómicas, de manera que familias que habitan en inmuebles sujetos a subsidios sean aquellas con menor capacidad económica, de acuerdo con lo señalado por la Corte Constitucional ${ }^{4}$, y aquellas con mayor capacidad de pago sean las que brinden una contribución mayor sobre las tarifas. En segundo lugar, proponiendo alternativas que procuren una sostenibilidad del sistema cruzado de subsidios, la utilización eficiente de recursos públicos y la incorporación de herramientas que hagan más progresivas las tarifas de $\mathrm{SPD}^{5}$.

Este libro presenta los principales resultados del estudio, reflexionando sobre varios puntos que se indagan a lo largo de los diferentes capítulos. ¿Responde la estratificación hoy en día al propósito de identificar (hogares en) inmuebles residenciales urbanos claramente diferenciados entre sí, que facilite la aplicación de subsidios y contribuciones en las tarifas de SPD? ¿Puede la información catastral captar de manera más precisa una realidad socioeconómica de los respectivos hogares, es decir, hay una mayor correlación de la información catastral con las características socioeconómicas de los hogares y con su capacidad de pago que aquella correlación que pueda existir con la estratificación? ¿Qué modelos alternativos de estratificación basados primordialmente en información catastral resulta en clasificaciones más adecuadas de los inmuebles residenciales que permitan una mejor focalización de la política de subsidios en SPD? ¿Existen las condiciones técnicas y la información suficiente para ir más allá de un modelo de clasificación de inmuebles por grupos y permitir una clasificación continua?

Es importante anotar que existe otro tipo de diagnósticos e intuiciones alrededor de la estratificación, que van más allá de las deficiencias técnicas y metodológicas, que este texto no aborda, pero que forman parte del debate de este instrumento de política pública. Entre los más destacados, se encuentra el impacto sobre incentivos a los agentes económicos (tanto hogares como empresas) en el consumo de los SPD, eficiencia de las empresas prestadoras

3 El concepto que incorpora la Ley 142 es el de 'inmueble residencial'.

4 "La clasificación de los usuarios en categorías, por sí misma, no viola la Constitución, siempre que la clasificación corresponda a niveles distintos de capacidad económica” (Sentencia C-252/97).

5 La progresividad se entiende como el atributo en las tarifas de SPD que hace que, estadísticamente, los predios que sean habilitados para recibir subsidios tengan usuarios de menor capacidad de pago, de forma tal que el porcentaje de gasto por SPD sea menor o equivalente al de usuarios con mayor capacidad de pago. 
de servicios o la posibilidad de generar (o ampliar) dinámicas de segregación o discriminación espacial urbana. Aunque dichas aristas del debate son importantes de analizar cuando se estudia el esquema de estratificación, no son el objeto de esta obra y no serán abordadas a lo largo de los capítulos ${ }^{6}$.

\subsection{Diagnóstico de la estratificación vigente: debilidades metodológicas, importantes errores de inclusión y riesgo de sostenibilidad del sistema}

La primera parte de este libro desarrolla un contexto general y un diagnóstico sobre estratificación socioeconómica en Colombia y presenta las posibilidades técnicas que ofrece la información catastral para la clasificación de viviendas en el Distrito Capital.

En el capítulo 2, Jaime Bonilla, Denis López y Carlos Sepúlveda exponen algunas debilidades metodológicas de la estratificación haciendo énfasis en los errores de inclusión y en el riesgo sobre la sostenibilidad financiera del sistema de subsidios cruzados en SPD.

Los autores buscan brindar una perspectiva general del balance de la estratificación socioeconómica en Colombia. Desarrollan, en primer lugar, un contexto básico sobre sus propósitos e implementación, y, a través de una revisión de literatura, presentan un diagnóstico en cuanto a su efectividad en su propósito de focalización de política de subsidios en las tarifas de SPD. Posteriormente, realizan una revisión sobre estudios que incorporan la información catastral como insumo alternativo para construir modelos de clasificación socioeconómica.

En el capítulo 3, Leonardo Bernal y Fabio Tejedor muestran cómo el catastro inmobiliario constituye un sistema integrado, que reúne sistemáticamente información técnica y objetiva para la totalidad de los predios de la ciudad, reflejando la diferenciación socioeconómica urbana y validando el carácter multifinalitario del catastro de Bogotá. Introducen de esta manera una variable central, el valor unitario integral (VUI), como medida referente

6 Santamaría et al. (2009) afirman que los subsidios cruzados afectan la competitividad de los sectores industriales y comerciales. Para una exposición adecuada respecto a la relación con discriminación o segregación, ver los estudios sobre segregación urbana desarrollados por la Secretaría Distrital de Planeación y la Universidad Nacional de Colombia (SDP-CID, 2014) y los estudios sobre desarrollo humano en Bogotá liderados por Jorge Iván González (PNUD, 2008, 2014). 
de la información catastral para las propuestas alternas a la estratificación socioeconómica. Esta variable es analizada por Acosta et al. en el capítulo 4 y hace comparable el valor de metro cuadrado de un predio de propiedad horizontal ( $\mathrm{PH}$ ) y uno de no PH, al consolidar en ella el área construida y el tamaño del terreno.

\subsubsection{DEBILIDADES METODOLÓGICAS E IMPORTANTES ERRORES DE INCLUSIÓN}

La metodología de estratificación vigente (administrada primero por el Departamento Nacional de Planeación -DNP- y, desde 2004, por el Departamento Administrativo Nacional de Estadística-DANE-) parte de un principio de objetividad, en la medida que se basa en características observables del inmueble a través de un procedimiento homogéneo y técnico que facilita la comparación. Sin embargo, luego de años sin actualizar, la metodología presenta limitaciones importantes:

1) variables de alta incidencia en la definición actual del estrato como las zonas hábitat son definidas de manera subjetiva (por ejemplo, conceptos como desviación social o sector residencial exclusivo);

2) dentro del modelo de determinación del estrato, no hay ponderación de variables por poder discriminante. Además, si bien la observación es a nivel de vivienda, en Bogotá, D.C., se consolida (calculando la moda) a nivel de manzana por predominancia de dicha variable, desconociendo la diversidad en el interior de cada una de ellas;

3) es vulnerable a presiones políticas (difícil actualización por eventuales costos políticos);

4) existen deficiencias en el flujo de información entre instituciones involucradas y falta de alineación en las prioridades de política que dificulta su seguimiento y mejora. Esto se ilustra y se refuerza con el hecho de que la metodología vigente no ha tenido ningún cambio estructural desde su implementación en 1994, a pesar de haber una mejora sustancial en los estudios de diagnóstico y los sistemas de información disponibles en Bogotá, D.C.

Con el paso de los años, el instrumento de estratificación dejó de reflejar la diferenciación de grupos a partir de su capacidad socioeconómica, 
evidente por los errores de inclusión significativos (hogares sin condiciones para ser subsidiados son clasificados en estratos bajos), que traen como consecuencia problemas de sostenibilidad del sistema. Esta desvinculación de la estratificación con la capacidad de pago de los hogares está acompañada con el hecho de que resulta imposible, en la situación actual de información (primaria o secundaria) con que cuenta el país, extraer información directa, confiable y estandarizada que permita clasificar la totalidad de los hogares según sus ingresos.

Pensando, por otro lado, en que la estratificación actual se concibe como clasificación de viviendas (inmuebles residenciales en el sentido de la Ley 142 de 1994), la mejor alternativa en este sentido es a través de la información que permite calcular el avalúo. No existen registros administrativos universales que ofrezcan suficientes elementos para estimar un conjunto adecuado de variables para diferenciar la capacidad de pago.

Teniendo en cuenta que la diferenciación socioespacial que se ejerce para la ocupación del suelo urbano en una economía de mercado es resultado de la diferenciación socioeconómica (expresada ella en ingresos o capacidad de pago), un desarrollo que involucre la información catastral (las zonas homogéneas físicas y las zonas geoeconómicas con todas sus variables) constituye información útil para sustentar una conceptualización y un modelo de la diferenciación por niveles de ingreso y calidad de vida. Esto se evidencia en el alto nivel de correlación entre las características de las viviendas, medidas a través de la información catastral (las variables de calificación de construcción y la determinación de zonas homogéneas físicas y geoeconómicas) y las condiciones socioeconómicas de los hogares.

El catastro ofrece un conjunto importante de variables que permiten caracterizar, diferenciar y agrupar los domicilios usuarios de SPD en grupos homogéneos (estratos) e, incluso, pensar adelante en una política de focalización y manejo de subsidios y contribuciones de SPD soportada directamente en la información catastral, sin necesidad de estratificar.

Las decisiones metodológicas parten de utilizar la mejor información disponible para lograr ese objetivo y el uso de la información catastral ha sido una alternativa natural dentro del debate acerca de las mejoras metodológicas que deben considerarse a futuro sobre clasificación socioeconómica. En un principio, la metodología de estratificación optó por una herramienta diferente ante evidencia de limitaciones en la actualización y sistematización. Sin 
embargo, las mejoras de catastro, especialmente para la ciudad de Bogotá, D.C., hacen que esta opción vuelva a estar presente y pase a ser técnicamente mucho más eficiente que la vigente.

\subsubsection{INFORMACIÓN CATASTRAL: FUENTE TÉCNICA, OBJETIVA Y SISTEMÁTICA PARA CLASIFICACIÓN DE INMUEBLES RESIDENCIALES}

Soportado en datos objetivos de los atributos asociados (jurídicos, físicos, de dotación de recursos y de valor comercial), el sistema de información predial es el inventario detallado de las características del suelo y las construcciones (aspecto físico), y la zonificación de áreas de homogeneidad física y económica sintetiza aquella diferenciación socioeconómica existente en lotes, construcciones, localizaciones y entornos en la ciudad. Este tema ha sido explorado por otros estudios, quienes han presentado evidencia sobre la utilidad y efectividad de la información catastral como base para rediseñar la estratificación socioeconómica o, si esta llegase a abandonarse, para clasificar a los usuarios de servicios públicos domiciliarios sobre los cuales se exigirán contribuciones o a quienes se les aportarán subsidios (Sardi, 2007, 2008; DANE, 2011).

El capítulo 3 presenta una conceptualización que sustenta que el valor (precio) de mercado de los inmuebles (cuya proxy es el avalúo catastral) sintetiza las diferencias existentes en las áreas urbanas, desde los aspectos físicos y el disfrute de bienes públicos, hasta los aspectos ideológicos (prestigio, estatus, autosegregación). La información que se levanta en los procesos catastrales constata la diferenciación existente en el tipo de viviendas que los usuarios de suelo habitacional adquieren según la calidad constructiva, el tipo de materiales utilizados, el área construida, el mobiliario y las características de sus acabados, la edad (vetustez) y el estado de conservación, que reflejan la capacidad económica que tienen sus propietarios u ocupantes para pagar por las calidades y comodidades que cada tipo de construcción ofrece, o, en la otra dirección, para renunciar a ellas y demandar viviendas de calidad inferior que se ajusten a su capacidad real de pago.

Desde una perspectiva analítica sobre la lógica de los mercados de tierras y los mecanismos de formación y realización y renta del suelo, se relacionan los diferenciales del avalúo catastral, como una proxy del precio del suelo urbano, con las condiciones socioeconómicas de quienes acceden a inmuebles. Con este enfoque analítico, Bernal y Tejedor buscan conectar las desigualdades en medidas socioeconómicas, como la capacidad de pago, con la posibilidad 
de acceder o no acceder y usufructuar determinada localización intraurbana, determinado tipo de vivienda y condiciones de hábitat relacionadas con el entorno urbanístico y la calidad de vida urbana, las cuales se diagnostican en este mismo capítulo como altamente desiguales. En esta línea, el capítulo 3 ilustra empíricamente la reconfiguración urbana de Bogotá, en términos de dinámica demográfica, diferenciales de calidad de vida urbana y diferenciación espacial en los valores catastrales, para introducir el fenómeno de diferenciación-fragmentación-segregación socioeconómica en función de la lógica del mercado de suelo urbano y de la capacidad de pago de los moradores que acceden a los distintos productos inmobiliarios.

En la formación de los avalúos inmobiliarios, se cristaliza, sintetiza y concretiza una parte fundamental de la diferenciación urbana. En el avalúo, se concentra el peso de diversas variables diferenciadoras de los entornos de la ciudad. Y, dado que tenemos un sistema de información en el que el valor de los inmuebles es una variable central, se entiende que utilizarlo para Bogotá, como base para diferenciar a los residentes urbanos, es técnicamente sustentable para definir ya sea los 'estratos' (6 o 150), o para que, eliminados los estratos, se administren los subsidios y las contribuciones de SPD.

Al proponer la información catastral como insumo principal para el cobro de tarifas públicas, Bernal y Tejedor estudian el efecto del estrato en el cálculo del avalúo actual y estiman un avalúo alternativo que elimine este efecto. Como es lógico, una alternativa futura a la estratificación no puede estar influenciada por la estratificación vigente. Finalmente, construyen el VUI, variable que hace comparable el valor de metro cuadrado de un predio de propiedad horizontal ( $\mathrm{PH}$ ) y uno de no $\mathrm{PH}$, al consolidar en ella el área construida y el tamaño del terreno. El vUI condensa aspectos relevantes del entorno y la construcción, lo que lleva a ser propuesto como elemento central en las alternativas de modelos de estratificación o esquemas diferentes de definición de subsidios cruzados de SPD en inmuebles.

\subsection{Modelos de estratificación socioeconómica con base en la información catastral: mejoras insuficientes}

Los capítulos 4 y 5 parten de la conceptualización presentada en el capítulo 3 y profundizan en un análisis estadístico que relaciona la información catastral con la de encuestas de hogares, y presentan propuestas de actualización de dicho esquema. 
En el capítulo 4, Javier Acosta, Alexis Maluendas y Guillermo Rivas profundizan desde el punto de vista estadístico en las hipótesis planteadas en los capítulos 2 y 3 . A partir de cinco índices que dan cuenta de la capacidad de pago y de las condiciones de vida de los hogares, confirman, en primer lugar, la problemática de errores de inclusión que presenta la estratificación vigente. En segundo lugar, ahondan en el estudio de la relación de las condiciones socioeconómicas de los hogares con la información catastral de los predios en que habitan. De manera particular, demuestran la estrecha correlación del valor unitario integral con los diferentes índices.

La identificación de estas relaciones permitirá más adelante proponer clasificaciones alternativas de los inmuebles residenciales. Una vez confirmada la correspondencia entre las características de los inmuebles y las condiciones socioeconómicas de los hogares que los habitan, se podrá incluir como insumo principal de la nueva propuesta metodológica de estratificación o clasificación de predios para el cobro de los SPD. Lo valioso de los ejercicios realizados por los autores es que permite un emparejamiento entre información de registro catastral y encuesta de hogares, como la Encuesta multipropósito para Bogotá 2011 (EMB 2011).

De esta manera, respecto a la problemática de la estratificación vigente, en línea a lo expuesto en el diagnóstico del capítulo 2, Acosta et al. muestran que, a pesar de existir tradicionalmente una correlación entre estrato y capacidad de pago (o ingreso) de los hogares, el instrumento no se ha ajustado adecuadamente a las dinámicas económicas y urbanas, y no refleja óptimamente las desigualdades socioeconómicas de la población desde distintas medidas (capacidad de pago, índices de bienestar, entre otros). Hogares en deciles altos de gasto o ingreso tienen una alta probabilidad de vivir en bienes inmuebles clasificados en estratos bajos (altos errores de inclusión). Específicamente, para hogares dentro de los primeros ocho deciles de capacidad de pago, existe una probabilidad significativamente mayor de habitar en un inmueble en estrato 2 o 3 que de ser clasificado en otro estrato. Hogares con alta capacidad de pago deberían, idealmente, contribuir al sistema (ser clasificados en estratos 4, 5 y 6), y están recibiendo subsidios.

Con respecto al segundo objetivo del capítulo 4, referente a la pertinencia de la información catastral, se muestra para Bogotá una fuerte relación entre la batería de indicadores socioeconómicos con las variables físicas y precios del suelo de los inmuebles residenciales. Particularmente, presenta evidencia 
del alto grado de asociación de las medidas propuestas con vUi y el puntaje de la construcción.

En línea con estos resultados, y a partir de las conclusiones respecto a la pertinencia de la información catastral en general y el VUI en particular, en el capítulo 5, Denis López y Carlos Sepúlveda exponen tres modelos alternativos de estratificación socioeconómica con base en la información de la ficha catastral vigencia 2012. El primero parte de un escenario nacional que considera un ajuste metodológico que involucra a todos los catastros del país y las diferentes entidades relacionadas del orden nacional y distrital. En este sentido, se hace uso de modelos de probabilidad que ponderan diferentes variables contenidas en la ficha catastral y asignan un estrato a cada predio residencial urbano para la ciudad ${ }^{7}$. La segunda alternativa implementa un modelo discriminante directamente (y exclusivamente) para el Distrito Capital a partir del vUI del avalúo) y sin tener en cuenta información de otros catastros. Estos dos modelos se desarrollan con base en la normatividad vigente en Colombia, es decir, se restringe la posibilidad de estratos a seis. El último modelo amplía la segunda opción, flexibilizando la norma y permitiendo que el número de estratos se determine endógenamente. En este caso, resulta un número óptimo de nueve estratos.

Los tres modelos propuestos se evalúan a partir de su impacto (frente al sistema vigente) respecto a los cambios de estrato de los diferentes inmuebles, a errores de exclusión e inclusión a partir de varios indicadores, y, finalmente, al impacto económico dentro del balance del sistema de subsidios cruzados. En este último punto, el impacto financiero mantiene los porcentajes de subsidio y contribuciones actuales, por lo que no se considera para la alternativa de nueve estratos. Ante una eventual implementación, tendrían que revaluarse por parte de las respectivas comisiones de regulación los rangos de subsidios cruzados establecidos por ley, de manera que, ante la nueva clasificación, se logre un diseño de tarifas más progresivo que el de las actuales.

Para los tres modelos, una nueva estratificación traería una mejor clasificación en términos de que una menor proporción de hogares con altos ingresos habitan viviendas clasificadas en estratos bajos, brindando una mejora notoria respecto a la sostenibilidad del sistema y a un mejor uso de

7 Estos modelos probabilísticos garantizan en pasos previos una correlación de estas variables catastrales con características socioeconómicas de los hogares y una diferenciación adecuada entre estratos. 
los recursos públicos. No obstante, a partir de los resultados del capítulo 5 , surgen varios dilemas. El escenario ideal que considera a toda la nación (y, por lo tanto, capta toda la diferenciación urbana del país) representa la alternativa que resulta en menores errores de inclusión, pero, a su vez, los cambios en los estratos asignados son tan drásticos para el Distrito Capital que, de mantenerse los porcentajes de subsidios y contribuciones actuales, sería impráctica su implementación. A manera de ilustración, los estratos 5 y 6 pasarían de representar el $9 \%$ de inmuebles en Bogotá al $43 \%{ }^{8}$. Este modelo, adicionalmente, resulta complejo de explicar debido a la multiplicidad de pasos metodológicos en su implementación, lo que llevaría a una dificultad pedagógica adicional. Finalmente, parte del supuesto de que la información entre los diferentes catastros del país es homogénea en términos de calidad, nivel técnico y sistematización, lo cual se aleja de la realidad.

El segundo modelo, por su parte, es un primer paso para explorar alternativas diferenciadas en Colombia en la administración de la política de tarifas de SPD. Debido a la dinámica y compleja evolución urbanística de Bogotá (y las principales ciudades capitales), y a la mejora de la información catastral del Distrito Capital frente a los demás catastros nacionales, es válido considerar opciones diferenciales en los métodos de estratificación. Esta opción sería un camino mucho más rápido y transparente de implementar (en términos pedagógicos, debido a la sencillez del modelo), pero intermedio (entre la estratificación vigente y la alternativa nacional) en términos de errores de inclusión. El último modelo, al definir nueve estratos, logra una mejora en la diferenciación de los grupos, pero sus implicaciones (respecto al esquema actual) no son drásticamente diferentes a lo encontrado con los otros dos modelos anteriores y requiere modificaciones estructurales a la normatividad actual.

Un cambio hacia los modelos, resultado de una aplicación exclusiva en el Distrito Capital, conlleva un escenario donde la probabilidad de ser asignado a un estrato más alto aumenta a medida que los deciles de capacidad de pago aumentan, y mejora con relación a la estratificación vigente. Es más probable para hogares de deciles bajos habitar inmuebles con asignación de estrato 1, y

8 Estas fuertes variaciones recogen dos aspectos. En primer lugar, el cambio metodológico que permite clasificar más adecuadamente los bienes inmuebles; y, en segundo lugar, la mejora en las condiciones de vida de los habitantes de la ciudad. 
para hogares en deciles altos habitar inmuebles de estrato 6. Estos resultados llevan a plantear una primera recomendación para el Distrito Capital:

Partiendo de un esquema de estratificación de bienes inmuebles para la definición de tarifas de SPD en el país y en el Distrito, la utilización de una metodología que tome el ava ún catastral como variable que lleve a la asignación de estratos, cuyo número no esté predeterminado (o mejor se calcule de manera endógena), resulta en una clasificación de inmuebles más efectiva, viable, transparente y clara para la ciudadanía y con mejoras sustanciales respecto a posibles errores de inclusión (sin corregirlos completamente).

Sin embargo, a pesar de la mejora en términos de eficiencia fiscal en la asignación de subsidios de SPD, la probabilidad de pertenecer a los estratos 2 y 3, dado el decil, sigue dominando a las demás probabilidades (aunque no de manera tan fuerte), desde el decil 1 al 8. Al ampliarse a nueve estratos, esa dominancia se corrige mucho más claramente, pero no de manera ideal. El hecho de que un eventual esfuerzo institucional para transitar a este nuevo esquema de estratificación presente aún errores de inclusión significativos lleva a pensar en propuestas de definición de sistemas impositivos cruzados en SPD más allá de la estratificación tradicional.

\subsection{Hacia un desmonte de la estratificación socioeconómica}

El sistema de estratificación es un caso atípico en el contexto internacional, y las ventajas que en su momento lo justificaron pierden vigencia hoy en día ante la posibilidad de utilizar directamente el avalúo catastral para determinar subsidios cruzados sin necesidad de establecer grupos diferenciados. Juan Miguel Gallego, Luis Hernando Gutiérrez, Denis López y Carlos Sepúlveda analizan, en el capítulo 6, el esquema de estratificación colombiano en el marco de la teoría económica de diseño de tarifas y a la luz de la experiencia internacional sobre subsidios en el sector. El análisis ubica el alcance de este libro dentro del contexto teórico y empírico en el mundo.

Dentro de un contexto de definición de cobros en la prestación de los SPD (monopolio natural), la estratificación segmentó el mercado en seis estratos, lo cual clasificó a los usuarios por (potencialmente) diferentes niveles de ingresos. Al clasificar los hogares (o sus viviendas) por grupos y establecer por unidad de consumo cobros iguales en el interior de los grupos pero diferentes entre grupos, la definición de pagos se acerca a una discriminación de precios 
de tercer grado. Adicionalmente, a nivel internacional, las herramientas de identificación de potenciales subsidiarios pasan desde la identificación de áreas, barrios o zonas geográficas específicas, a la identificación de hogares directamente a partir de registros poblacionales o de su inscripción a programas sociales específicos.

Al preguntarse por la eficiencia en el esquema de pagos de SPD derivada de la estratificación, Gallego et al. presentan ciertas observaciones. Por un lado, la estratificación ha sido efectiva en el cumplimento del propósito de cobertura en SPD, llegando en muchos casos al 100\% en las principales ciudades del país. Por otro lado, sin embargo, al ser regresivo, el esquema no permite una redistribución adecuada del ingreso. Por último, hoy en día, las condiciones técnicas y la disponibilidad de información alternativa permiten plantear la discusión de reformular el esquema, moviéndose de la estratificación de inmuebles a un tipo de discriminación de primer grado, que defina el pago en los servicios públicos domiciliarios directamente a partir del avalúo catastral. Esto lleva a tener un punto intermedio entre el ideal, de identificación directa de hogares potenciales beneficiarios de subsidios (y contribuciones) en SPD, a uno que tome provecho de un registro de información técnico y actualizado sobre sus viviendas.

Desde el punto de vista de focalización del gasto público y particularmente de los subsidios a los hogares, es importante contar con herramientas complementarias de información y no pretender basarse en un instrumento único de identificación (de hogares). Problemas en el desarrollo del instrumento SISBEN (Sistema de Identificación de Potenciales Beneficiarios de Programas Sociales), por ejemplo, ante el cual los hogares presentan comportamientos estratégicos para ser incluidos en el programa, alertan sobre el cuidado de migrar rápidamente hacia un esquema único de registro de hogares. Adicionalmente, el estado actual de este tipo de registros no permite implementar esquemas cruzados de subsidios, al no ser universales. De ahí la utilidad de tener un instrumento disponible y robusto, como la información catastral, para canalizar este tipo de subsidios en SPD.

En esta línea, el capítulo 6 realiza un ejercicio exploratorio en busca de una mayor eficiencia fiscal, que fortalezca el sistema solidario y redistributivo de tarifas de SPD, y presenta una primera propuesta de determinación de subsidios cruzados en SPD basada en un escenario hipotético en que no utilice la concepción de estratos socioeconómicos o clasificación de grupos 
(de viviendas u hogares) y determine un esquema ligado al respectivo avalúo catastral de los bienes inmuebles. El ejercicio busca ilustrar una manera sencilla de establecer el cobro de los SPD directamente del avalúo catastral e iniciar la discusión sobre el tema.

A partir de un propósito de 'equiesfuerzo', en el que todos los hogares resulten con una misma proporción de gasto en el SPD respecto a su gasto total, y se garantice así el balance para la empresa de servicio público (cubrimiento del costo medio), Gallego et al. brindan un esquema sencillo de determinación de subsidios cruzados para el servicio de acueducto, que se determina directamente del avalúo catastral del respectivo inmueble sin necesidad de confirmar estratos y resulta en pagos crecientes por unidad de consumo (metro cúbico) a medida que el avalúo aumente. Esto corrige hasta cierto punto el problema de regresividad del sistema actual, y muestra que una mejor aproximación a las características de la vivienda (por el avalúo) resulta en una disminución en el pago de facturas (respecto a la percibida actualmente) para los deciles 1 al 7 y un aumento no muy pronunciado de la factura mensual para los deciles $9(\$ 11.500)$ y $10(\$ 41.000)$. Cabe aclarar y resaltar que, al ser un ejercicio exploratorio, no se tienen en cuenta cambios en el consumo de los hogares ante el cambio de montos en la factura, elemento que debe considerarse en un estudio de mayor profundidad. Adicionalmente, a futuro, debe depurarse la propuesta de forma que no resulte en un subsidio a los consumos totales de servicios públicos, sino que garantice el pago de consumos básicos de subsistencia. La propuesta debe de esta manera armonizarse con ajustes a la estructura tarifaria, para que contenga tarifas diferenciales por consumo e incorpore elementos como la composición y el número de personas en el hogar, el impacto del consumo suntuario en el gasto total de servicios públicos, las costumbres relacionadas con el consumo de cada hogar, entre otros.

Ciertas preguntas quedan abiertas: una vez abordado el tema redistributivo a través del avalúo catastral, ¿cuáles son los impactos sobre el consumo del servicio? ¿Qué incentivos (positivos y negativos) se generan entre los diferentes agentes del mercado? ¿Qué tipo de políticas de tarifas diferenciadas se pueden establecer que garanticen la eficiencia económica por servicios particulares? ¿Cuál sería el impacto de este esquema en los precios de la tierra, la vivienda y los arriendos? 


\subsection{Recomendaciones}

Los diferentes capítulos desarrollados en este libro llevan a plantear recomendaciones puntuales sobre el desarrollo de la política en el diseño de subsidios cruzados y tarifas en servicios públicos domiciliarios.

\subsubsection{ESQUEMAS ALTERNATIVOS A LA ESTRATIFICACIÓN ACTUAL}

La estratificación socioeconómica en Colombia, y de manera particular en Bogotá, requiere de una revisión metodológica de fondo que va desde cambios en la información básica hasta su misma existencia. Esta revisión debe considerar varios puntos:

Los ejercicios estadísticos y econométricos presentados en esta obra son evidencia para que el Distrito Capital, y en general el gobierno nacional, considere la información catastral, y en particular el avalúo, como una herramienta técnica pertinente para el diseño de subsidios cruzados y tarifas de servicios públicos domiciliarios. Hoy en día, el Distrito Capital cuenta con un catastro que ha hecho esfuerzos importantes para llegar a una actualización catastral anual y de calidad que permite acceder a la mejor información disponible sobre bienes inmuebles en la ciudad. El operativo de estratificación resulta en un esquema de recolección de información paralelo a catastro, costoso y técnicamente subóptimo.

El marco normativo actual requiere ajustes importantes. Manteniendo el sistema de estratificación socioeconómica, debe permitirse que el número de estratos no esté predeterminado en seis, sino que se establezcan endógenamente a partir de la diversidad de las características de los bienes inmuebles por estratificar en un momento dado. Esto lleva a implementar varios procesos (jurídicos, tecnológicos e interinstitucionales) que requieren una agenda pública amplia.

La discusión de fondo que resulta de las conclusiones de este libro pone en consideración la posibilidad de plantear una política de tarifas de SPD que desmonte la estratificación socioeconómica en Bogotá D.C. A pesar de su innegable pertinencia y del impacto que jugó en la política pública, este esquema único en el contexto internacional parece perder relevancia en las últimas décadas, debido a los problemas que genera sobre la eficiencia del gasto público, desfase presupuestal importante a nivel nacional y problemas de focalización de subsidios para los hogares más necesitados. Adicional- 
mente, su característica de herramienta transparente y sencilla pierde fuerza ante la disponibilidad de, cada vez mejor, información oficial de los hogares y sus viviendas.

El Distrito Capital presenta una fortaleza en su información catastral que permite poder evolucionar a esquemas técnicamente más eficientes y establecer subsidios cruzados en SPD a partir del avalúo catastral directamente, sin necesidad de estratificar. Es decir, transitar de un sistema de facturación por grupos (estratos) hacia uno que refleje mejor las condiciones socioeconómicas de cada hogar/vivienda (considerando el avalúo como proxy de la capacidad de pago de los hogares). Esta transición sería ideal para todo el país, pero, debido a las fortalezas institucionales del Distrito, es mucho más factible para la capital en el corto plazo. Es importante que el esquema por considerar sea lo suficientemente flexible y dinámico, de manera que no resulte en derechos adquiridos indefinidamente, que pueda fortalecerse, actualizarse y adaptarse a dinámicas urbanas y poblacionales específicas y a disponibilidad de información adicional pertinente.

Es cierto que un esquema ideal debería buscar focalizar subsidios y contribuciones directamente en los hogares, a partir de su capacidad de pago. Esto puede lograrse a través de varios instrumentos: declaración de renta universal, registros administrativos de hogares e individuos, modelos de aproximación a partir de encuestas, entre otros. Ninguno de los primeros están disponibles en la actualidad y aquellos instrumentos existentes (e. g., encuesta SISBEN) tienen problemas similares de inclusión y universalidad. Modelos de aproximación a partir de encuestas es un mecanismo inferior al propuesto en esta publicación. A pesar de que el país debe procurar fortalecer continuamente la información de los hogares y los diferentes registros administrativos que capten su información, una política de focalización de subsidios debe incorporar instrumentos alternativos y complementarios que brinden información completa de los hogares. De ahí la importancia de potenciar la información catastral para este fin. También es cierto que el instrumento catastral no es perfecto. Por un lado, la relación entre el avalúo de los bienes inmuebles y la capacidad de gasto de los hogares se rompe en casos específicos, como en proyectos de renovación urbana focalizados en mejoras de vivienda de hogares con muy baja capacidad de pago o ante la presencia de fenómenos de pobreza oculta, en que sectores con un alto nivel de calidad de vida urbana y desarrollo humano histórico está ocupado por familias que van perdiendo 
su poder adquisitivo y su capacidad de pago ${ }^{9}$. Para ambos casos, se requieren instrumentos específicos de excepción y políticas complementarias focalizadas. Por otro lado, modelos basados en el avalúo de la vivienda pueden verse afectados por factores especulativos, muchos de ellos derivados de fenómenos externos, 'extraurbanos', que pueden impactar directamente los avalúos (precios) sin que ello signifique necesariamente un cambio en las características socioeconómicas o de ingreso de la población que se localiza en el espacio urbano.

\subsubsection{REQUERIMIENTO DE MEJORAS CONTINUAS DE LA INFORMACIÓN CATASTRAL Y MIGRAR HACIA UN CATASTRO MULTIFINALITARIO}

Independientemente de los caminos que decida la administración pública nacional y distrital frente al futuro de la estratificación socioeconómica, al transitar a un escenario donde la información catastral es la base fundamental para la clasificación de inmuebles residenciales, se requiere una serie de acciones tendientes a mejorar dicha información:

a) Modificar la ficha predial, pues tanto los ítems que contiene como los materiales y métodos constructivos y niveles conservativos a los que se refiere (y que se traducen en puntajes de materiales y conservación) están en desuso. Corresponden a condiciones de hace 25 años y no incorporan los cambios de materiales y métodos de construcción o conservación desarrollados desde entonces.

b) La determinación de las zonas físicas homogéneas, con base en criterios más amplios. Hasta hoy, se utilizan: accesibilidad a vías, topografía, infraestructura y cobertura de servicios, modelo constructivo (condominios, casas unifamiliares, edificios, etc.), normatividad proveniente de instrumentos de planificación vigentes, pero se dejan por fuera otros elementos que constituyen el paisaje físico de las ciudades (características sociogeográficas, características arquitectónicas de los

9 En Bogotá, proyectos de renovación urbana, como Fenicia, alrededor de la Universidad de los Andes (http://www.eltiempo.com/Multimedia/especiales/feniciarenovacionbogota/fenicia-la-revolucionurbana-del-centro_13613235-7), y casos puntuales en Teusaquillo (http://www.elespectador.com/ noticias/bogota/pobreza-oculta-de-bogota-articulo-499963), ilustran ambos ejemplos. 
domicilios por separado y en conjunto, características urbanísticas, disponibilidad y disfrute de bienes públicos, características económicosociales, mezcla de usos, exclusividad, conjuntos cerrados, etc.) y entorno socioambiental (tanto negativo, como focos de contaminación, vertimiento, islas de calor etc., como positivos, isótopos de reflexión, zonas verdes, áreas de producción de oxígeno, barreras naturales y artificiales, etc.).

c) Eliminar, al menos de forma explícita, el efecto del estrato en la determinación del avalúo catastral, más específicamente en la estimación de los valores unitarios de construcción (teniendo en cuenta los resultados del impacto del estrato en el avalúo).

d) Recomendar la evaluación de la pertinencia de incluir algunas de las variables asociadas a la determinación de precios del suelo en el establecimiento de los valores unitarios de construcción (valor por metro cuadrado del terreno, variables asociadas a las zonas físicas homogéneas, variables de ubicación en la ciudad, etc.).

La nueva posibilidad de uso de la información catastral hace que la comprensión de los objetivos, estructura, naturaleza, insumos y productos, fines y medios del catastro se ajusten hacia una perspectiva de un sistema amplio e integral de información multifuncional, generando importantes avances en la calidad de los sistemas de información sobre tierras. Esto implica un cambio paulatino de paradigma que garantice mejor calidad en la información y más amplios elementos sobre los que deba producirse tal información, a fin de servir a la ciudad en sus distintas dependencias para definir políticas públicas de intervención en el territorio y de planificación del desarrollo urbano en todas sus acepciones, facetas, componentes, procesos y actuaciones.

La racionalidad detrás del catastro multifinalitario es que no solo es posible, sino necesario que el gobierno local logre, continúe y acelere el proceso dinámico de los últimos años, que resulte en información cada vez más objetiva, técnica y detallada no solo sobre los inmuebles y no solo para propósito fiscal (catastro tradicional), sino también sobre el territorio (características urbanísticas, geográficas, arquitectónicas, entornos diferenciados y focos de influencia positiva o negativa), de manera que pueda conformar extensas y relevantes bases de datos que incluyen, además de los ya convencionales datos inmobiliarios (datos jurídicos, físicos, geométricos y de avalúos de los 
predios), información sobre las características demográficas, sociales, económicas y ambientales que permiten conocer a profundidad ya no el predio, sino el territorio y sus características, en las que se ubican los predios y de quienes los habitan o les dan uso. Ello permite vincular la información a un mapa de parcelas en un sistema de información geográfica (SIG) que da lugar a una administración eficiente de la información útil ya no solo para definir políticas fiscales, sino todo tipo de políticas de intervención y planeación sobre el territorio. 